Im Journal Club dieser Ausgabe der pädiatrie hautnah referieren und kommentieren diese Experten für Sie Arbeiten aus der internationalen Fachliteratur.

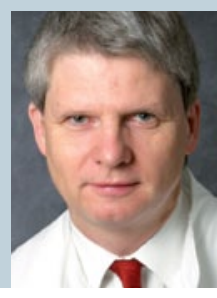

Prof. Dr. med. Dietrich Abeck, München

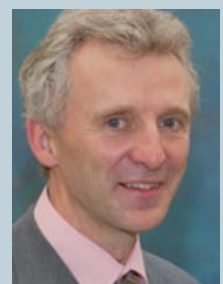

Dr. med. Martin Claßen, Bremen

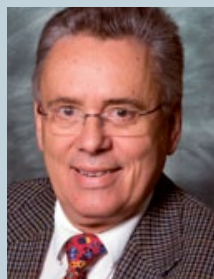

Dr. med. Hartmut Koch,

Vechta

\title{
Blastocystis bei Bauchschmerz
}

Die Bedeutung von Blastocystis hominis für rezidivierende Bauchschmerzen bei Kindern wird immer wieder diskutiert. Ein Team aus der Schweiz konnte nun zeigen, dass die Protozoen keine Auswirkungen haben.

Rezidivierende Bauchschmerzen bei Kindern und Jugendlichen sind in einem hohen Prozentsatz durch Infektionen oder ein postenteritisches Reizdarmsyndrom bedingt. Insofern gehört

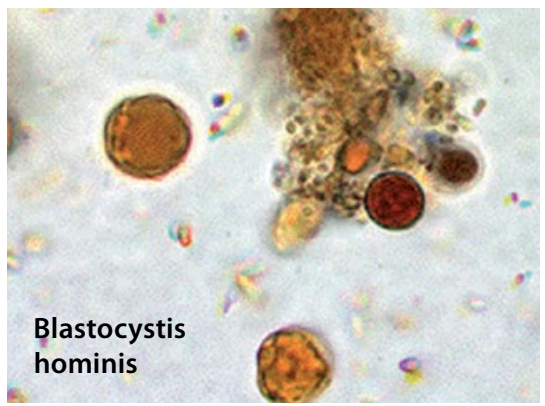

die Infektionsdiagnostik - auch im Hinblick auf Protozoen - zur Standarddiagnostik. In den Stuhlproben wird immer wieder Blastocystis hominis nachgewiesen. Dann stellt sich aber das Problem, dass die Pathogenität dieses Erregers bislang nicht als eindeutig bewiesen gilt.

Eine Gruppe aus Zürich hat nun bei 40 Kindern mit rezidivierenden Bauchschmerzen und Blastocystis-hominisNachweis im Stuhl eine prospektive, doppelblinde und placebokontrollierte Therapie mit Cotrimoxazol versus Placebo durchgeführt. In der CotrimoxazolGruppe beendeten 20 die Studie, in der Placebo-Gruppe 17. In beiden Gruppen verminderten sich die Schmerzindizes: In der Verum-Gruppe von 6,9 auf 4,1 und unter Placebo von 7,4 auf 3,0. Dieser Unterschied war nicht signifikant. Zudem gab es zwischen dem Verschwinden der Keime und der Besserung der Beschwerden keine Korrelation.

Heyland K et al. No advantage for antibiotic treatment over placebo in blastocystis hominis-positive children with recurrent abdominal pain. J Pediatr Gastroenterol Nutr 2011; Oct 12 [Epub ahead of print]

Kommentar: Schon immer war die Pathogenität von Blastocystis hominis sehr umstritten. Die aktuelle Studie unterstützt die Einschätzung, dass ein Blastocystis-Nachweis durchaus ignoriert werden darf. Darüber hinaus zeigt sich ein sehr guter Placeboeffekt. Trotzdem wird man sich in Einzelfällen für eine antibiotische Therapie entscheiden. Cotrimoxazol ist dabei eine genauso gute Wahl wie das bisher verwendete Standardantibiotikum Metronidazol. Dr. Martin Claßen

\section{Selbstbeschädigung mit Selbstheilungstendenz}

Insgesamt 1.943 australische Jugendliche im mittleren Alter von knapp 16 Jahren wurden zwischen 1992 und 2008 regelmäßig befragt. Besonderes Augenmerk wurde auf selbstverletzendes Verhalten der Jugendlichen und dessen Entwicklung im Laufe der Jahre gelegt.

$\mathrm{D}$ ie Selbstbeschädigung wird als eine Handlung mit nicht fatalem Ergebnis definiert, mit der Absicht, sich selbst zu schaden. Das kann vom Zufügen von Verletzungen bis hin zur Einnahme von toxischen Substanzen oder Fremdkörpern reichen. Es handelt sich um einen der stärksten Prädiktoren eines Suizids. Besonders häufig ist das Verhalten bei Frauen in der Altersgruppe von 15-24 Jahren zu finden. Eine populationsbasierte Studie zur Selbstbeschädigung hatte es bislang noch nicht gegeben. Paul Moran und Kollegen holten dies nun nach: Zwischen 1992 und 2008 wurden in geschickt ausgewählten Stichproben die Jugendlichen und jungen Erwachsenen in neun Intervallen mit einjährigem Abstand befragt. Erst drei Jahre nach

$8 \%$ der Adoleszenten fügen sich selbst Verletzungen zu, bei jungen Erwachsenen liegt die Rate nur noch bei $1 \%$.
Beginn der Befragungen wurde erstmals nach Selbstbeschädigung gefragt. Von den Teilnehmern waren 1.802 in der Adoleszenz.

$10 \%$ der Mädchen und $6 \%$ der Jungen berichteten, dass sie bei sich schon Selbstbeschädigungen vorgenommen

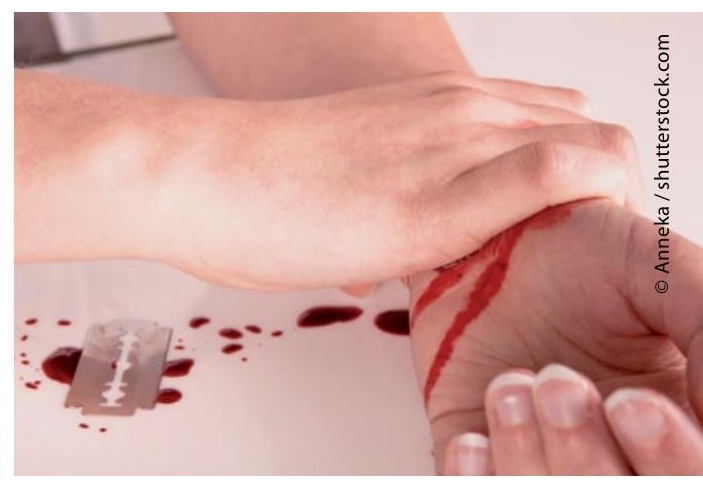

